\title{
Calculation of low bandgap homopolymers: Comparison of TD-DFT methods with experimental oligomer series
}

\author{
Eliezer Fernando Oliveira a , Juan Carlos Roldao ${ }^{a}$, Begoña Milián-Medina ${ }^{\mathrm{b}, \mathrm{c}}$, \\ Francisco Carlos Lavarda ${ }^{\mathrm{a}, \mathrm{d}}$, Johannes Gierschner ${ }^{\mathrm{c}, *}$ \\ a UNESP - Univ Estadual Paulista, POSMAT - Graduate Program in Material Science and Technology, Av. Eng. Luiz Edmundo Carrijo Coube 14-01, \\ 17033-360 Bauru, SP, Brazil \\ ${ }^{\mathrm{b}}$ Department for Physical Chemistry, Faculty of Chemistry, University of Valencia, Avda. Dr. Moliner 50, 46100 Burjassot, Valencia, Spain \\ ' Institute for Advanced Studies, IMDEA Nanoscience, C/Faraday 9, Campus Cantoblanco, 28049 Madrid, Spain \\ ${ }^{d}$ DF-FC, UNESP - Univ Estadual Paulista, Av. Eng. Luiz Edmundo Carrijo Coube 14-01, 17033-360 Bauru, SP, Brazil
}

\section{A R T I C L E I N F O}

\section{Article history:}

Received 29 October 2015

In final form 29 December 2015

Available online 6 January 2016

\begin{abstract}
A B S T R A C T
The performance of different DFT functionals (B3LYP, BHLYP, CAM-B3LYP, M06HF) on the prediction of vertical transition energies $E_{\text {vert }}$ of low bandgap homopolymers is tested against the experimentally available oligomer series (thienopyrazines and thienothiophenes). This allows for a detailed and accurate comparison on the consistency of DFT methods for chainlength evolution and polymer limit prediction, and for an understanding of geometry and time-dependent contributions to $E_{\text {vert }}$ by combinatorial analysis. Together with former studies on wide/medium bandgap polymers and low bandgap co-polymers, our results on low bandgap homopolymers suggest offset-corrected M06HF as the most viable method for time inexpensive and reliable prediction of semiconducting polymers at the moment.
\end{abstract}

(c) 2016 Elsevier B.V. All rights reserved.

\section{Introduction}

Conjugated low bandgap polymers have been the target of intense studies in the past for their promising performance in organic photovoltaics and field effect transistors [1-8]. In fact, plastic-based optoelectronic devices have several advantages compared to their inorganic counterparts, including low cost, flexibility, transparence, easy processing, and improved sustainability, but especially the tunability of their electronic and optical properties via versatile organic synthesis. However, versatility has its price, requiring tedious efforts in synthesis and characterization to explore the properties of new polymers. Thus, with the rise of cost-effective quantum-chemical methods and fast and cheap computational resources, polymer property prediction prior to synthesis has found much interest in the community [9-11].

However, reliable polymer bandgap prediction is still a challenge due to the large number of atoms, which cannot be accessed with the most accurate of today's methods. Thus, most polymer calculations are based on (time-dependent) density functional theory, (TD)DFT, but the proper choice of the functional to reliably calculate (optical) bandgaps is in dispute. In fact, the most employed

\footnotetext{
* Corresponding author.

E-mail address: johannes.gierschner@imdea.org (J. Gierschner).
}

B3LYP functional for this purpose suffers from a number of pitfalls $[12,13]$, which result in an incorrect evolution in bond-length alternation (BLA) [14-16], ionization potentials [17], and electronic transitions $[9,18]$ as a function of system size, as well an overestimation of molecular orbitals (MO) delocalization [19]. It was sufficiently stressed before, that such consistency of the geometrical, electronic and optical properties with the size of the conjugated system is mandatory to avoid correct results for wrong reasons by error compensation $[9,18]$. Such chainlength consistency tests for a given polymer have thus to be done before doing polymer prediction via periodic boundary calculations or via the oligomer approach. Thus, the only way to accurately test DFT functionals is a careful comparison with the available experimental data of the electronic and optical properties of the oligomers $[9,18]$; however, taking care that calculated values are properly compared with experiment. This includes the choice of an appropriate theoretical extrapolation method to extract the polymer limit values, as well as misleading comparisons between the vertical ( $\left.E_{\mathrm{vert}}\right)$ and adiabatic transition energies ( $E_{00}$; i.e. the optical bandgap). Other contributions which directly affect the experimental data are thermal, substituent, solvent and solid state effects [9].

The number of available experimental studies on the optical properties of oligomers series, which enable such comparison are, however, limited. In fact, only on wide and medium bandgap homopolymers a considerable number of studies are found in 
literature, mainly based on (substituted) phenylene, thiophene, fluorene repetition units (RU) as well as their vinylene- or ethynylene-linked variants (see, e.g. the reviews in Refs. [9,20,21]). For low bandgap materials, only a few donor-acceptor co-oligomer [22,23] and homo-oligomer [24-26] series were reported.

For a number of medium bandgap homopolymers and the few existing low bandgap co-polymers, Wykes et al. [18] have performed such comparative TD-DFT studies on oligomers of different length to test the consistency of the method with the chainlength evolution of $E_{\text {vert }}$. The studies reproduced the overestimation of the chainlength evolution by B3LYP, but also showed that longrange correction (LC) by optimally tuned LC-BLYP did not lead to great improvement; this problem was also addressed elsewhere [27], and it was suggested that explicit solvation gives much better agreement with experiment [28]. Improvement against B3LYP was obtained by long-range corrected CAM-B3LYP [18]. The main parameter for an improvement was however the increase of Hartree-Fock (HF) exact exchange (EXX), stressing the importance of such contributions also at the short range [27]. M06HF (100\% EXX) showed a consistent evolution with chainlength compared to experiment [18] favored by the correct representation of the asymptotic behavior of the potential [27]. Nevertheless, an (expected) overestimation of the absolute energies is observed. It was thus suggested to introduce an offset correction (OC) of $-0.75 \mathrm{eV}$ to the calculated vertical energies (in vacuum) to compare with the experimental ones (in solution) [18]. Although solvent shifts $\Delta E_{\text {solv }}$ are of considerable importance [9], they are fairly constant with chainlength for longer oligomers (i.e. $\geq$ two repetition units) [29,30], and even when comparing different oligomer series; i.e. a bathochromic shifts of $\Delta E_{\text {solv }} \approx-0.3 \mathrm{eV}$ is induced [9]. Similar considerations apply to thermal effects; going from the $T=0 \mathrm{~K}$ limit to room temperature, hypsochromic shifts of $\Delta E_{\text {therm }} \approx 0.15 \mathrm{eV}$ are found for systems with shallow ground state torsional potentials $[9,30]$. An unbiased implicit treatment of solvent and temperature effects in TD-DFT is possible [31], requires however specialized knowledge and testing, and it is thus not suitable for routine polymer prediction. Therefore, OC-M06HF in vacuum was suggested as a feasible strategy for rapid and at the same time reliable estimation of $E_{\mathrm{vert}}$ in solution for medium bandgap homo- and low bandgap co-polymers [18].

Nevertheless, for low bandgap homopolymers, a similarly designed accurate test is missing by now. These polymers are expected to be particularly challenging from a theoretical view due to quinoid contributions [32,33]. We will meet this challenge in the present letter, performing TD-DFT at different levels of theory on the (only) experimentally available oligomer series of low-bandgap polymers. The latter are thieno[3,4-b]pyrazines ( $n$ TP; where $n=$ is the number of RU) with two kind of end terminations [24,25], and thienothiophenes ( $n$ TT) [26]. Furthermore, combinatorial analysis of mixed B3LYP/M06HF calculations will provide an understanding of geometry and TD contributions to the evolution of $E_{\text {vert }}$ with chainlength.

\section{Materials and methods}

Experimental $E_{\text {vert }}$ were taken as absorption band maxima ${ }^{1}$ of thienopyrazine polymer (PTP) [34] as well as oligomers ( $n$ TP; with $n=1,2,3$ ), end-terminated with trimethylsilyl (TMSnTP) [25] and thiophene (TnTP) [24] groups. A second series of compounds were thienothiophene oligomers with (2,4,6-triisopropylphenyl) end groups ( $\mathrm{Pr}_{3} \mathrm{PnTT}$ ) with $n=1-6$ [26]. Solvents for the experimental

\footnotetext{
1 This procedure to extract $E_{\mathrm{vert}}$ is only approximate [9], however the error is expected to be smaller than $0.05 \mathrm{eV}$.
}

spectra were chloroform for TP, TMSnTP, and Pr3PnTT and dichloromethane for TnTP.

Ground state geometries were fully optimized by DFT with various functionals, including B3LYP with 20\% EXX [35-37] and dispersion-corrected B3LYP-D3(BJ) [38], BHLYP (50\% EXX) [36], M06HF (100\% EXX) [39] and the long-range corrected CAM-B3LYP [40] and wB97XD functionals [41]. The $n$ TP oligomers were found to be all co-planar in anti-conformation; the same co-planar conformation was as well observed between the T and TP units in TnTP oligomers. ${ }^{2}$ An anti-conformation was also found for the $\mathrm{Pr}_{3} \mathrm{PnTT}$ oligomers, however slightly twisted by ca. $10^{\circ}$ between the TT units, whereas the substituted phenyl groups are strongly twisted against the backbone (ca. 80-90 ${ }^{\circ}$ ). To avoid unsystematic noise in the TD calculations for different oligomer length, the TT units were kept co-planar for the following TD calculations. Vertical absorption transition energies $E_{\text {vert }}$ were calculated by TD-DFT using the functionals mentioned above. All (TD-)DFT calculations were done in vacuum in the $T=0 \mathrm{~K}$ limit employing the $6-31 \mathrm{G}^{* *}$ basis set as described in the Gaussian 09 program package [42].

Within the oligomer approach, the electronic and optical properties of the polymers are estimated through extrapolation from the oligomers results, mostly done either by an (empirical) exponential 3-parameter fit as introduced by Meier et al. [43], or by the (physically founded) coupled oscillator 2-parameter model proposed by Kuhn [44], as reviewed earlier [9,18]. In the Kuhn equation,

$$
E(n)=E_{1} \sqrt{1-D_{\mathrm{k}} \cos \left(\frac{\pi}{N+1}\right)}
$$

$E$ represents the transition energy of the oligomer, $N$ is the number of double bonds of the shortest path within the TP and TT backbone, respectively (i.e. not counting the bonds in the terminal thiophene and phenylene units), $E_{1}$ is the transition energy of a formal double bond $(N=1)$, and $D_{\mathrm{k}}=2\left(k_{\mathrm{s}} / k_{\mathrm{d}}\right)$ is a relative force constant $(k)$ that measure how strong the double bonds (d) are coupled by a single bond (s). However, as pointed out earlier [9], fits according to the Kuhn or Meier equation may be deficient if strong terminal effects are present; this might particular be true for oligomers with stronger quinoid contributions, since those will depend critically on the system size. We therefore use an extension of the original Kuhn formula by an exponential term to provide a better adjustment to experiment (i.e. modified Kuhn; MK), as shown in Eq. (2) [9].

$E(N)=E_{1} \sqrt{1-D_{\mathrm{k}} \cos \left(\frac{\pi}{N+1}\right)}-A e^{-B N}$

\section{Results and discussion}

The experimental chainlength evolution of $E_{\mathrm{vert}}$ for the TMS- and T-terminated $n$ TP oligomers, as well as for the $\mathrm{Pr}_{3} \mathrm{P} n \mathrm{TT}$ oligomers in solution are shown in Figure 1 [24-26]. $E_{\text {vert }}$ of the T-terminated TP monomer (T1TP) is much smaller compared to TMS1TP, due to the strongly enlarged conjugation length by the presence of the thiophene end groups in TnTP. However, the extrapolated polymer value is very similar in both series due to the vanishing effect of the terminating groups with increasing chainlength, resulting in $E_{\text {vert }}$ of around $1.2 \mathrm{eV}$ at the polymer limit. Here, the difference between TMS- vs. T-termination is ascribed to the rather large error in the extrapolation of only three experimental oligomer values. The reported experimental polymer (PTP) value in chloroform

\footnotetext{
2 A torsional scan was performed for $\mathrm{H}-$, Th-, and TMS-terminated TP dimers $(n=2)$ giving a planar anti conformation. Oligomers with up to $12 \mathrm{RU}$ were fully optimized; no imaginary frequencies were found.
} 


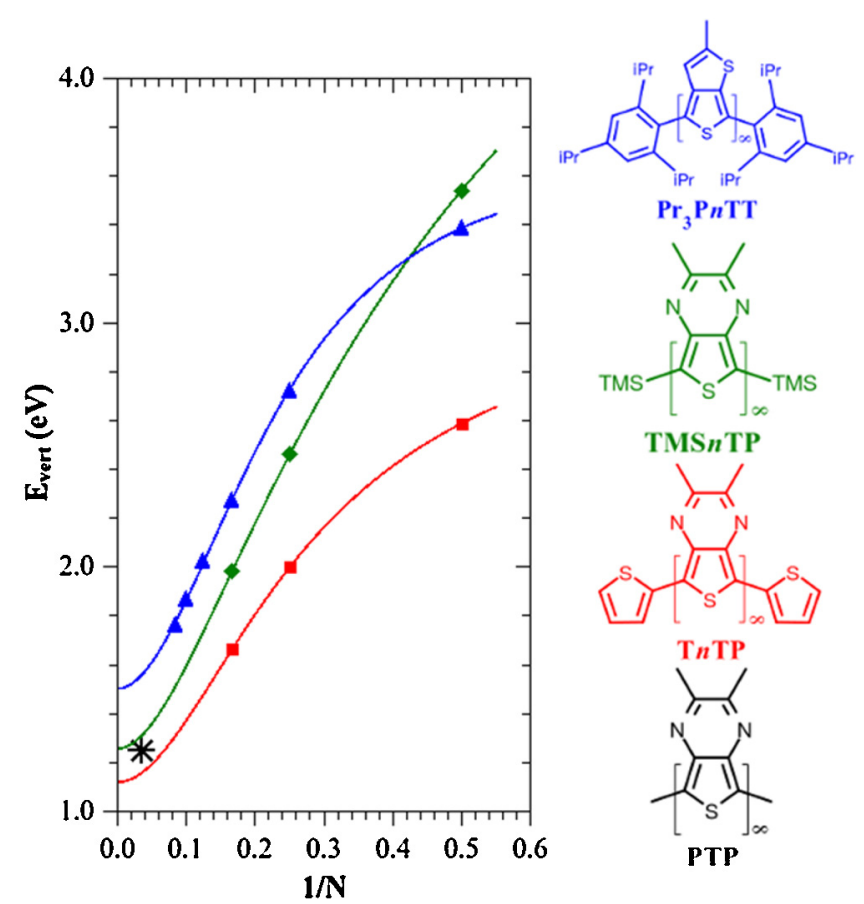

Figure 1. Experimental chainlength evolution of vertical transition energies $E_{\mathrm{vert}}$ of thienopyrazine based oligomers TMSnTP (green diamonds), TnTP (red squares) $[22,23]$ and thienothiophene $\operatorname{Pr}_{3}$ PnTT (blue triangles) oligomers [25]; the polymer value of PTP (black star) is shown for comparison. Lines are MK fits according to Eq. (2).

solution is $1.25 \mathrm{eV}$ [34], which agrees quite well with the extrapolation results taking into account that the molecular weight of the reported PTP is about $4300 \mathrm{~g} / \mathrm{mol}$, which corresponds to about 14 monomeric units. The polymer value of the $n$ TT series is estimated to be $1.5 \mathrm{eV}$. The strong change in the slope when going from $n=1$ to $n=2$ in $n$ TT is again ascribed to an end-effect, i.e. by the presence of the terminal phenyl rings. Apart from that, the chainlength evolution of the $n$ TT series is similar to the $n$ TP series; here, the overall higher values of $E_{\text {vert }}$ for the $n$ TT series reflect the less electron-poor nature of the TT unit as compared to TP.

In a first step of the calculation, we did the geometry optimization (GO) and the time-dependent (TD) part (i.e. to calculate $E_{\text {vert }}$ ) with the same functional, comparing the results with the available experimental data. Figure 2 presents the results for $E_{\text {vert }}$ of the TMSnTP series, while the results on TnTP are found in the supplementary information (SI). As expected, B3LYP only provides reasonable results for small to medium sized oligomers, but strongly overestimates the slope of the chainlength evolution, so that the polymer limit is close to $0 \mathrm{eV}$, more than $1 \mathrm{eV}$ below the experimental value of $1.2 \mathrm{eV}$. It should be noted that the underestimation of the polymer value is much larger than what is seen in thiophene-based medium bandgap homopolymers and low bandgap co-polymers [18]; the reasons will be discussed later in this letter. Anyway, we want to stress again at this point that our results show that B3LYP allows not even for clear trends among different polymer series. Dispersion correction via B3LYP-D3(BJ) hardly improved the result (see SI). As for the systems discussed by Wykes et al. [18], CAM-B3LYP and BHLYP give similar results, providing a noticeable improvement against B3LYP but still overestimating the slope and thus giving transition energies below $1 \mathrm{eV}$. The dispersion and long-range corrected wB97XD functional only slightly improves the results against CAM-B3LYP, see SI. M06HF shows, as expected, a strong hypsochromic shift against experiment, but we note a very similar chainlength evolution as in

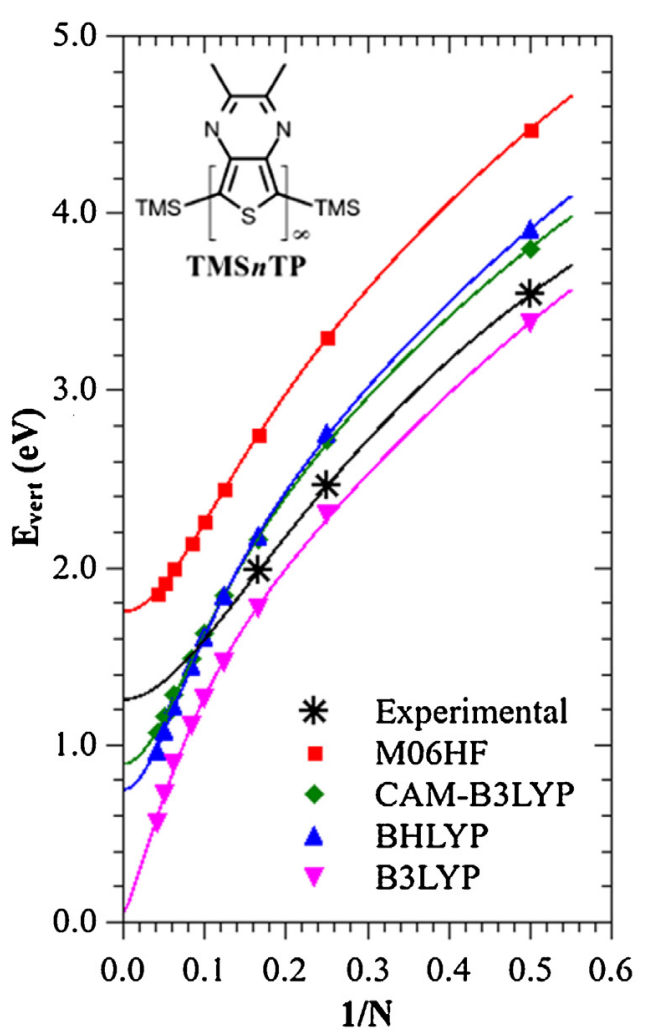

Figure 2. Vertical transition energies $E_{\text {vert }}$ for TMSnTP oligomers calculated with different (TD)DFT methods in vacuum (GO and TD part done with the same functional); experimental results (in solution) are shown for comparison.

experiment; this is consistent with previous findings [18], that the increase of EXX from B3LYP (20\%), BHLYP (50\%) to M06HF (100\%) significantly improves the results. The same trends were observed for the T-terminated, TP oligomers, and for TT oligomers, see SI

Due to the good performance of M06HF in the consistency with chainlength evolution on wide/medium bandgap homopolymers and low bandgap co-polymers, it was proposed earlier to use an offset correction (OC) of $-0.75 \mathrm{eV}$ to compare the M06HF results properly with experiments [18]. As seen in Figure 3, we can fully confirm this result for both TMS- and T-terminated TP series, as well as for the TT series, giving a remarkable agreement of OC-M06HF for the chain evolution compared with experiment. Hence, together with the former results by Wykes et al. [18], OC-M06HF can be indeed recommended as a viable method for time inexpensive and reliable calculations of vertical transition energies of wide to low bandgap homo- and co-polymers.

It should be reminded in this context that low bandgap oligomers are particularly challenging for theory due to the quinoid contributions which are reflected in the increase of the coupling between the double bonds, expressed in $D_{\mathrm{k}}$ of the Kuhn expression, see Eq. (1). For TMSnTP this gives $D_{\mathrm{k}}=0.92$ as compared to $D_{\mathrm{k}}=0.89$ for the oligothiophene $(n \mathrm{~T})$ series, but similar to the $n$ TTP co-oligomers $\left(D_{\mathrm{k}}=0.93\right)$ [18]. The remarkably strong deviation of B3LYP against experiment for the $n$ TP series (vide supra) should be related to its quinoid character, which offers here a chance to trace back the B3LYP failure in detail. To do this, a combinatorial analysis on the GO and TD parts of the (TD)DFT calculations, i.e. TD//GO was performed, where the M06HF and B3LYP functionals were employed. As can be seen from Figure 4, the mixed situations M06HF//B3LYP and B3LYP//M06HF lie in between the pure M06HF and B3LYP ones; however showing that the main reason for the strong failure of the B3LYP functional is found in the GO rather 


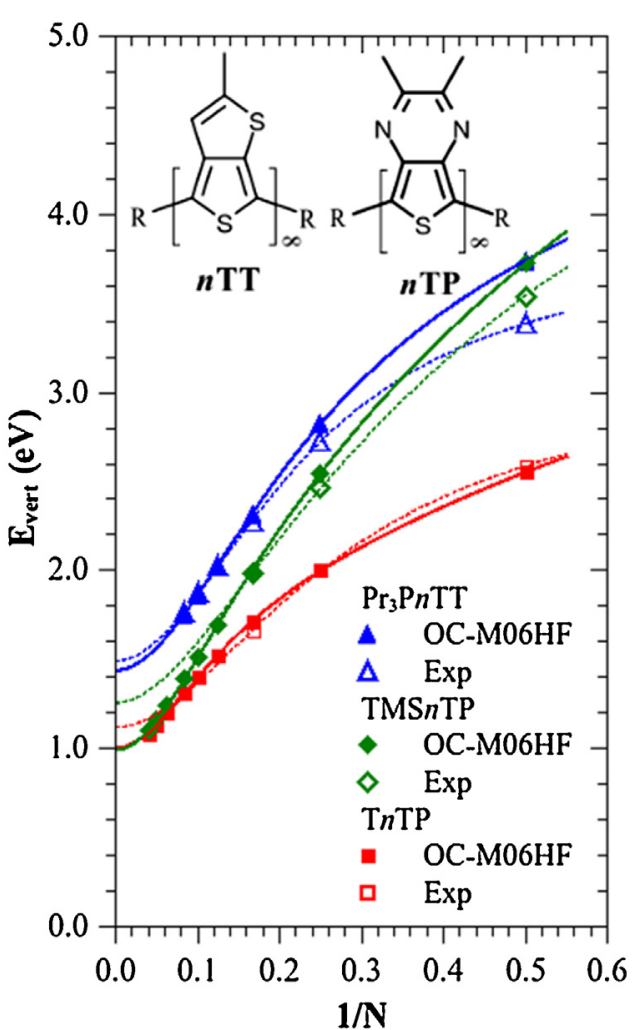

Figure 3. Vertical transition energies $E_{\text {vert }}$ for TMSnTP, TnTP and $\operatorname{Pr}_{3}$ PnTT oligomers: comparison of experimental and OC-M06HF $(\mathrm{OC}=-0.75 \mathrm{eV})$.

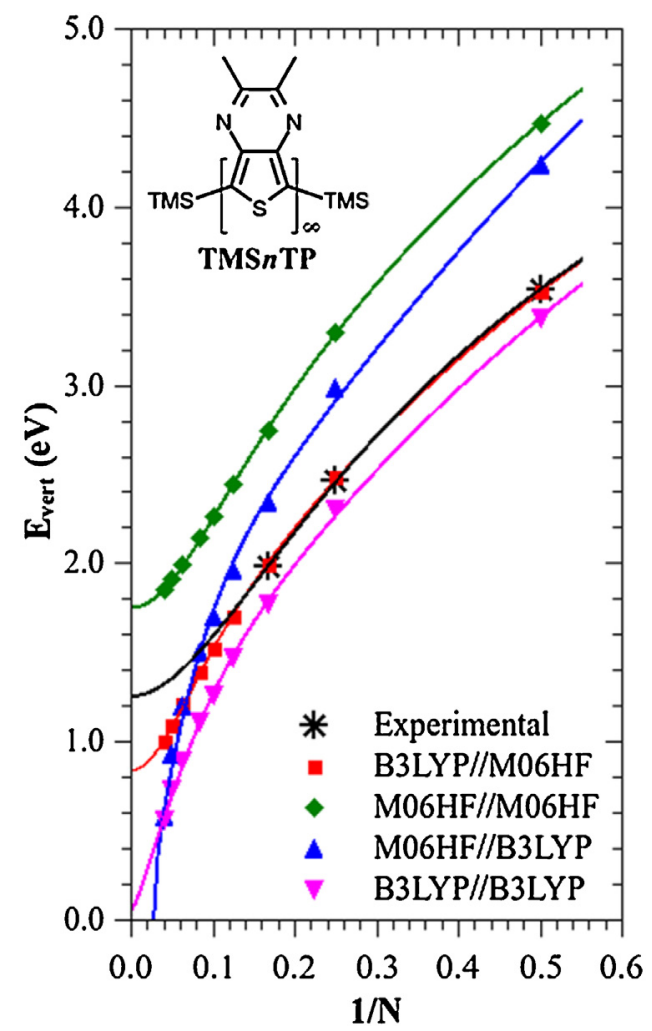

Figure 4. Combinatorial analysis of TMSnTP for all TD//GO combinations employing the B3LYP and M06HF functionals.
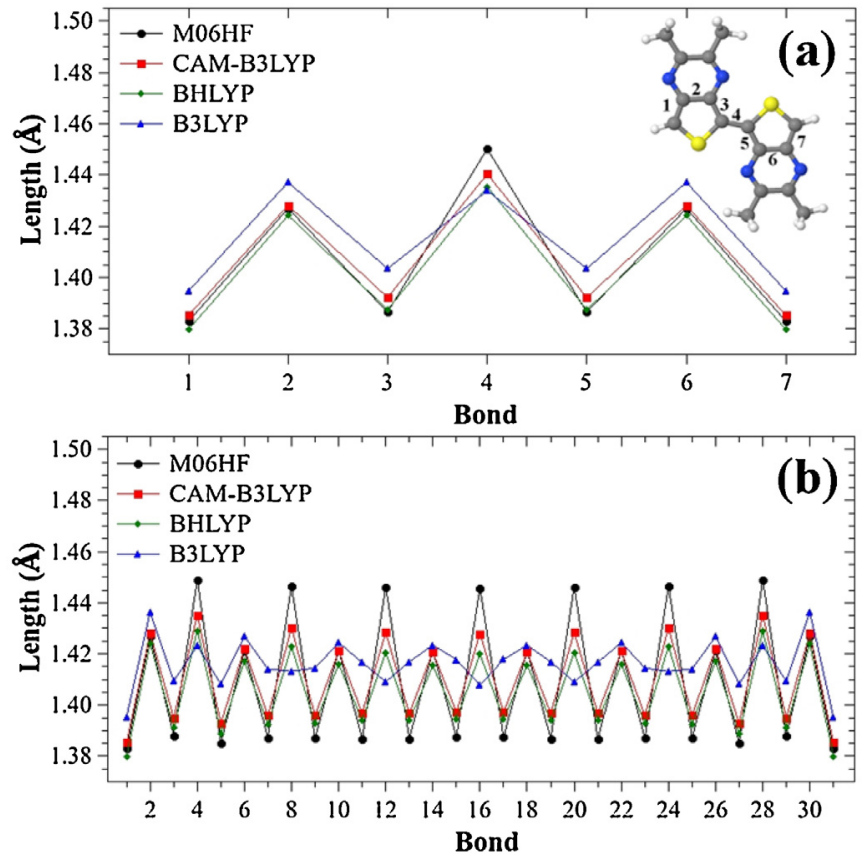

Figure 5. Bond lengths for (a) two and (b) eight monomeric units of TMSnTP along the conjugated path.

than in the TD part, leading to a sharp decrease of $E_{\mathrm{vert}}$ for long oligomers. $^{3}$

To further quantify the GO contribution, the bond length alternation (BLA) for short and long oligomers (two and eight RUs) of the TMSnTP series was analyzed by following the bond length along the conjugated path, see Figure 5. For short oligomers, the BLA increases essentially with the fraction of exact HF exchange in the functional as noted before [15], however the differences are not very pronounced. This explains why $E_{\text {vert }}$ of the short oligomers in Figure 4 are very similar if the TD part is the same (e.g. B3LYP//M06HF vs. B3LYP//B3LYP). On the other hand, for long oligomers, the bond lengths evolves dramatically different for B3LYP with respect to the other functionals, giving a very small BLA in the center of the molecule, very similar to what was reported for benzothiophenes [45]; this behavior clearly indicates that B3LYP geometries are not reliable for long low bandgap oligomers. It is the apparent reason for the large difference between M06HF//B3LYP and M06HF//M06HF in Figure 4, where the strong underestimation at the polymer limit is a direct result of the low BLA in B3LYP. Analogous results are obtained for the TnTP series, see SI.

\section{Conclusions}

In the current letter, we have performed a computational study on the experimentally available oligomer series of low bandgap homopolymers (thienopyrazines $n \mathrm{TP}$, and thienothiophenes $n \mathrm{TT}$ ) to test the performance of different DFT functionals (B3LYP, BHLYP, CAM-B3LYP, M06HF) on vertical transition energies $E_{\mathrm{vert}}$; this included both the consistency with chainlength as well as the behavior with respect to the absolute energies.

A systematic improvement in the chainlength consistency is found by increasing the amount of Hartree-Fock exact exchange

\footnotetext{
3 The B3LYP//M06HF results seem to adjust well with experiment, but for long oligomers a systematic underestimation is seen. However, doing the same evaluation for $\mathrm{H}$ - and Th-terminated structures (see $\mathrm{SI}$ ), it is noted that the B3LYP//M06HF are in disagreement even for small oligomers, indicating that the good results for TMS-terminated small oligomers was a coincidence by error compensation.
} 
(EXX) in the functional. Our combinatorial analysis of the geometry and time-dependent part shows that the failure of methods with small EXX such as B3LYP lies mainly in the strong underestimation of the bond length alternation; this becomes especially pronounced for the here investigated low bandgap homopolymers with enlarged quinoid contributions. Offset-corrected OC-M06HF $(\mathrm{OC}=-0.75 \mathrm{eV})$ is shown to be a viable method to reproduce the chainlength evolution of $E_{\text {vert }}$ of low bandgap homopolymers. Keeping in mind, however, the tendency of HF for over-localization, the good performance of HF might be partially due to error compensation. Nevertheless, together with the former study on wide/medium bandgap polymer and low bandgap co-polymers [18], OC-M06HF seems to be in the moment the best recommendation for a general time-inexpensive and reliable prediction of $E_{\mathrm{vert}}$ for semiconducting polymers.

\section{Acknowledgments}

We would like to thank the Brazilian agencies FAPESP (proc. 2012/21983-0 and 2014/20410-1) and CAPES for financial support. This research was also supported by resources supplied by the Center for Scientific Computing (NCC/GridUNESP) of the São Paulo State University (UNESP). The work at IMDEA and University of Valencia was supported by the Spanish Ministerio de Economía y Competitividad (MINECO; coordinated project MultiCrom, grant no. CTQ2014-58801), and by the Campus of International Excellence (CEI) UAM + CSIC.

\section{Appendix A. Supplementary data}

Supplementary data associated with this article can be found, in the online version, at doi:10.1016/j.cplett.2015.12.059.

\section{References}

[1] P.M. Beaujuge, C.M. Amb, J.R. Reynold, Acc. Chem. Res, 43 (2010) 1396.

[2] H.J. Son, F. He, B. Carsten, L. Yu, J. Mater. Chem. 21 (2011) 18934.

[3] P.-L.T. Boudreault, A. Najari, M. Leclerc, Chem. Mater. 23 (2011) 456.

[4] H. Zhou, L. Yang, W. You, Macromolecules 45 (2012) 607.

[5] Y. Li, Acc. Chem. Res. 45 (2012) 723.

[6] E. Zhou, K. Hashimoto, K. Tajima, Polymer 54 (2013) 6501.

[7] K. Takimiya, I. Osaka, M. Nakano, Chem. Mater. 26 (2014) 587.

[8] L. Dou, Y. Liu, Z. Hong, G. Li, Y. Yang, Chem. Rev. 115 (2015) 12633.

[9] J. Gierschner, J. Cornil, H.-J. Egelhaaf, Adv. Mater. 19 (2007) 173.

[10] S.S. Zade, N. Zamoshchik, M. Bendikov, Acc. Chem. Res. 44 (2011) 14

[11] C. Risko, M.D. McGehee, J.L. Brédas, Chem. Sci. 2 (2011) 1200.
[12] A. Dreuw, M. Head-Gordon, J. Am. Chem. Soc. 126 (2004) 4007.

[13] M. Hellgren, E.K.U. Gross, Phys. Rev. A 85 (2012) 022514.

[14] D. Jacquemin, A. Femenias, H. Chermette, I. Ciofini, C. Adamo, J.M. Andre, E.R. Perpète, J. Phys. Chem. A 110 (2006) 5952.

[15] J.C. Sancho-García, A.J. Pérez-Jiménez, Phys. Chem. Chem. Phys. 9 (2007) 5874.

[16] T. Körzdörfer, R.M. Parrish, J.S. Sears, C.D. Sherrill, J.L. Brédas, J. Chem. Phys. 137 (2012) 124305

[17] T. Körzdörfer, J.S. Sears, C. Sutton, J.L. Brédas, J. Chem. Phys. 135 (2011) 204107.

[18] M. Wykes, B. Milián Medina, J. Gierschner, Front. Chem. 1 (2013) 35.

[19] B. Milián-Medina, J. Gierschner, WIREs Comput. Mol. Sci. 2 (2012) 513.

[20] K. Müllen, G. Wegner (Eds.), Electronic Materials: The Oligomer Approach, Wiley-VCH, Weinheim, 2008.

[21] H. Meier, Angew. Chem. Int. Ed. 44 (2005) 2482

[22] B.P. Karsten, L. Viani, J. Gierschner, J. Cornil, R.A.J. Janssen, J. Phys. Chem. A 112 (2008) 10764

[23] B.P. Karsten, R.A.J. Janssen, Macromol. Chem. Phys. 212 (2011) 515.

[24] B.P. Karsten, R.A.J. Janssen, Org. Lett. 10 (2008) 3513.

[25] L. Wen, C.L. Heth, S.C. Rasmussen, Phys. Chem. Chem. Phys. 16 (2014) 7231.

[26] F. Liu, G.L. Espejo, S. Qiu, M. Moreno Oliva, J. Pina, J.S. Seixas de Melo, J. Casado, X. Zhu, J. Am. Chem. Soc. 137 (2015) 10357.

[27] T.B. de Queiroz, S. Kümmel, J. Chem. Phys. 141 (2014) 084303.

[28] T.B. de Queiroz, S. Kümmel, J. Chem. Phys. 143 (2015) 034101

[29] J. Gierschner, H.-G. Mack, L. Lüer, D. Oelkrug, J. Chem. Phys. 116 (2002) 8596.

[30] J. Gierschner, H.-G. Mack, H.-J. Egelhaaf, S. Schweizer, B. Doser, D. Oelkrug, Synth. Met. 138 (2003) 311.

[31] R. Improta, F.J. Avila Ferrer, E. Stendardo, F. Santoro, Chem. Phys. Chem. 15 (2014) 3320 ,

[32] M. Kertesz, C.H. Choi, S. Yang, Chem. Rev. 105 (2005) 3448.

[33] B.P. Karsten, L. Viani, J. Gierschner, J. Cornil, R.A.J. Janssen, J. Phys. Chem. A 11 (2009) 10343.

[34] L. Wen, B.C. Duck, P.C. Dastoor, S.C. Rasmussen, Macromolecules 41 (2008) 4576.

[35] A.D. Becke, J. Chem. Phys. 98 (1993) 1372

[36] A.D. Becke, J. Chem. Phys. 98 (1993) 5648.

[37] P.J. Stephens, F.J. Devlin, C.F. Chabalowski, M.J. Frisch, J. Phys. Chem. 98 (1994) 11623.

[38] S. Grimme, S. Ehrlich, L. Goerigk, J. Comput. Chem. 32 (2011) 1456.

[39] Y. Zhao, D.G. Truhlar, J. Phys. Chem. A 110 (2006) 13126

[40] T. Yanai, D. Tew, N. Handy, Chem. Phys. Lett. 393 (2004) 51.

[41] J.-D. Chai, M. Head-Gordon, Phys. Chem. Chem. Phys. 10 (2008) 6615.

[42] M.J. Frisch, G.W. Trucks, H.B. Schlegel, G.E. Scuseria, M.A. Robb, J.R. Cheeseman, G. Scalmani, V. Barone, B. Mennucci, G.A. Petersson, H. Nakatsuji, M. Caricato, X. Li, H.P. Hratchian, A.F. Izmaylov, J. Bloino, G. Zheng, J.L. Sonnenberg, M. Hada, M. Ehara, K. Toyota, R. Fukuda, J. Hasegawa, M. Ishida, T. Nakajima, Y. Honda, O. Kitao, H. Nakai, T. Vreven, J.A. Montgomery Jr., J.E. Peralta, F. Ogliaro, M. Bearpark, J.J. Heyd, E. Brothers, K.N. Kudin, V.N. Staroverov, R. Kobayashi, J. Normand, K. Raghavachari, A. Rendell, J.C. Burant, S.S. Iyengar, J. Tomasi, M. Cossi, N. Rega, J.M. Millam, M. Klene, J.E. Knox, J.B. Cross, V. Bakken, C. Adamo, J. Jaramillo, R. Gomperts, R.E. Stratmann, O. Yazyev, A.J. Austin, R. Cammi, C. Pomelli, J.W. Ochterski, R.L. Martin, K. Morokuma, V.G. Zakrzewski, G.A. Voth, P. Salvador, J.J. Dannenberg, S. Dapprich, A.D. Daniels, Ö. Farkas, J.B. Foresman, J.V. Ortiz, J. Cioslowski, D.J. Fox, Gaussian 09 - Revision D.01, Gaussian, Inc., Wallingford, CT, 2009.

[43] H. Meier, U. Stalmach, H. Kolshorn, Acta Polym. 48 (1997) 379

[44] W. Kuhn, Helv. Chim. Acta 31 (1948) 1780.

[45] J. Kürti, P.R. Surján, J. Chem. Phys. 92 (1990) 3247. 